\title{
Investigation of interaction of hydrogen with defects in zirconia
}

\author{
O. Melikhova ${ }^{1}$, J. Kuriplach ${ }^{1}$, J. Cizek ${ }^{1}$, I. Prochazka ${ }^{1}$, G. Brauer ${ }^{2}$, and W. Anwand ${ }^{2}$ \\ ${ }^{1}$ Charles University, Faculty of Mathematics and Physics, Department of Low Temperature \\ Physics, CZ-18000 V Holešovičkách 2, Prague, Czech Republic \\ ${ }^{2}$ Institut für Strahlenphysik, Forschungszentrum Dresden-Rossendorf, Postfach 510119, \\ D-01314 Dresden, Germany
}

\begin{abstract}
In the present work we study theoretically hydrogen incorporated into several positions in the zirconia cubic and tetragonal lattices. These are positions in the interstitial space and in the zirconium vacancy $\left(V_{\mathrm{Zr}}\right)$. We examine the structure of such configurations and for $V_{\mathrm{Zr}}$-related defects we also calculate selected positron characteristics in order to assess their capability of trapping positrons. It is shown that hydrogen atoms do not prefer to stay in the center of the largest interstitial space nor of $V_{\mathrm{Zr}}$ and they rather tend to create bonds with neighboring oxygen atoms. The positron lifetime of the $V_{\mathrm{Zr}}+1 \mathrm{H}$ complex is shorter than that for non-decorated $V_{\mathrm{Zr}}$ and positron trapping in $V_{\mathrm{Zr}}+1 \mathrm{H}$ complexes could, in principle, explain experimental lifetime data.
\end{abstract}

\section{INTRODUCTION}

Zirconia $\left(\mathrm{ZrO}_{2}\right)$ based materials are currently employed in a wide range of industrial applications (like refractories, grinding, solid oxide fuel cells), and many applications are yet under development. At ambient pressure zirconia exhibits three crystallographic phases: monoclinic, tetragonal and cubic. But many applications require the cubic phase that is not stable below $\sim 1400{ }^{\circ} \mathrm{C}$. Therefore, a stabilization of this cubic phase is needed, which is often done by additions of yttria $\left(\mathrm{Y}_{2} \mathrm{O}_{3}\right)$. The resulting material is then called yttria stabilized zirconia (YSZ). Due to stabilization, a large amount of oxygen vacancies $\left(V_{\mathrm{O}}\right.$ 's, charge +2$)$ must be introduced to compensate yttrium ions $\left(\mathrm{Y}^{3+}\right)$ that are negatively charged with respect to the lattice. Consequently, there are two $\mathrm{Y}$ ions per one $V_{\mathrm{O}}$ and corresponding defect complexes are denoted as $V_{\mathrm{O}}-2 \mathrm{Y}$ (or $\mathrm{Y}_{\mathrm{Zr}}^{\prime} \mathrm{V}_{\mathrm{O}}^{\bullet \bullet} \mathrm{Y}_{\mathrm{Zr}}^{\prime}$ in the Kröger-Vink notation). Thus, structural properties of YSZ materials are closely related to the composition of defects and their structure.

Positron annihilation (PA) techniques [1] are sensitive to open volume defects and can certainly help in defect identification in YSZ. The positron lifetime that is often measured in PA experiments depends on the size of defects, i.e. increases with the defect size. But the defect charge state also plays a role and positively charged defects, including oxygen vacancies, repulse positrons and cannot trap them. Conversely, neutral and negatively charged defects may act as positron traps provided that the corresponding positron potential well is deep enough. In the case of the $V_{\mathrm{O}}-2 \mathrm{Y}$ complex, which was considered to be the main positron trap in YSZ [2], our calculations [3] indicate that this complex does not constitute a positron trap (the corresponding positron potential well is not sufficiently deep). 
Besides oxygen vacancies and more complicated $V_{\mathrm{O}}-2 \mathrm{Y}$ complexes there might be other point defects in $\mathrm{ZrO}_{2} / \mathrm{YSZ}$. As hydrogen is a ubiquitous element and we have found recently that it is present in a large amount (of the order of 0.1 at.\%) in a variety of $\mathrm{ZnO}$ materials [4], we suppose that it should also exist in zirconia. Indeed, first measurements using nuclear reaction analysis indicate that YSZ materials contain an appreciable amount of hydrogen [5] comparable to that in $\mathrm{ZnO}$. Thus, a question arises in which positions hydrogen atoms can be located in the zirconia lattice and whether $\mathrm{H}$ atoms can interact with other defects existing in the $\mathrm{ZrO}_{2}$ lattice, which we investigate theoretically in this work though we present our first, partial results only.

In $\mathrm{ZnO}$ hydrogen may occupy interstitial positions [6] and/or may be present in the form of $\mathrm{H}_{2}$ molecules in 'channels' along the $c$-axis of the hexagonal lattice [6], the latter being the largest interstitial space in the lattice. Furthermore, hydrogen atoms can enter open volume defects, like oxygen and zinc vacancies and related defects (see e.g. [4,7,8]). Hydrogen may also form complexes with other impurities. It might be instructive to compare $\mathrm{ZnO}$ and $\mathrm{ZrO}_{2}$ cases.

As for $\mathrm{ZrO}_{2}$, the largest interstitial space in the cubic $\mathrm{ZrO}_{2}$ lattice is represented by a cube having eight $\mathrm{O}$ atoms in its corners $\left(\mathrm{O}_{8}\right.$ cube; see figure 1a), which we shall investigate first concerning $\mathrm{H}$ incorporation. We also study $\mathrm{Zr}$ vacancies as there are indications that $\mathrm{H}$ could reside in them, based on previous PA results [9]. It is interesting to note that the first neighbors of the $\mathrm{Zr}$ vacancy form also an $\mathrm{O}_{8}$ cube like for the interstitial space mentioned above, but the second nearest neighbors are different (figure 1c). Our recent work [9] contains very first results concerning $\mathrm{H}$ incorporation into the cubic $\mathrm{ZrO}_{2}$ phase. At the first stage of our investigations we do not consider the presence of $\mathrm{Y}$ atoms/ions in our calculations.
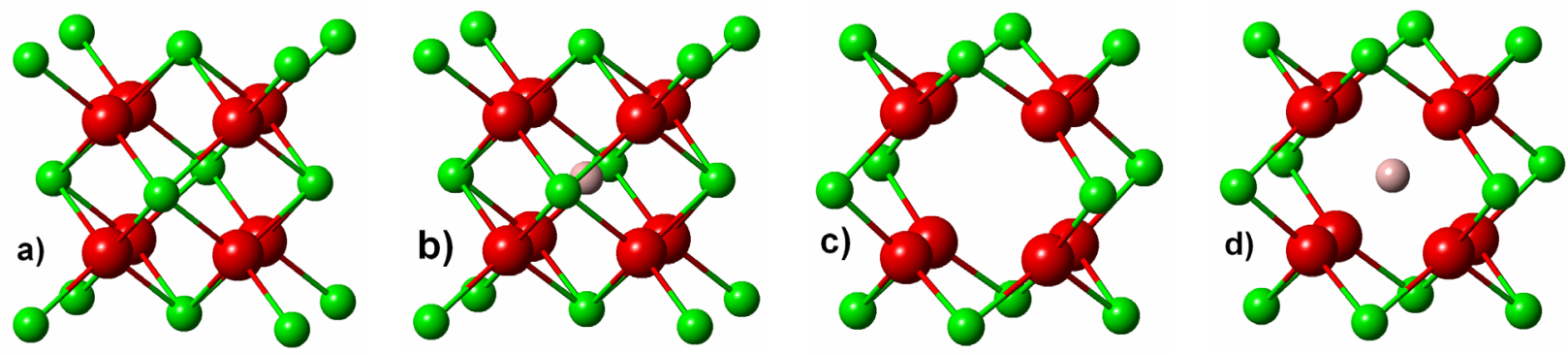

Figure 1. Cubic $\mathrm{ZrO}_{2}$ : a) perfect lattice, b) $\mathrm{H}$ in the $\mathrm{O}_{8}$ interstitial space, c) $V_{\mathrm{Zr}}$, and d) $\mathrm{H}$ in the center of $V_{\mathrm{Zr}}$. Red, green, and pink spheres represent, respectively, $\mathrm{O}, \mathrm{Zr}$, and $\mathrm{H}$ atoms.

\section{COMPUTATIONAL METHODS}

Realistic configurations of studied defects were obtained by means of relaxing the total energy of corresponding supercells with respect to atomic positions. In particular, the Vienna ab initio simulation package (VASP) [10] was employed for this purpose. In the course of calculations, projector augmented-wave potentials within the generalized gradient approximation were used [11]. The plane-wave energy cutoff was $400 \mathrm{eV}$ and a $2 \times 2 \times 2 \mathbf{k}$-space ( $\Gamma$ centered) sampling was selected. 96 atom-based supercells containing defects were utilized. The relaxation was stopped when forces at atoms fell below $0.01 \mathrm{eV} / \AA$. In this work the neutral charge state of defects was considered only.

The cubic $\mathrm{ZrO}_{2}$ phase (12 atom unit cell) studied here is shown in figure 1a. Furthermore, the tetragonal $\mathrm{ZrO}_{2}$ phase was also considered (figure $2 \mathrm{a}$ ). In the case of the cubic phase the symmetry was fixed to 'cure' its instability [12]. In addition to perfect cubic and tetragonal lattices we examined several configurations with $\mathrm{H}$ incorporated into the lattice. Namely, we 
placed an $\mathrm{H}$ atom into the interstitial $\mathrm{O}_{8}$ space and also into the zirconium vacancy and relaxed atomic coordinates as described above.

a)

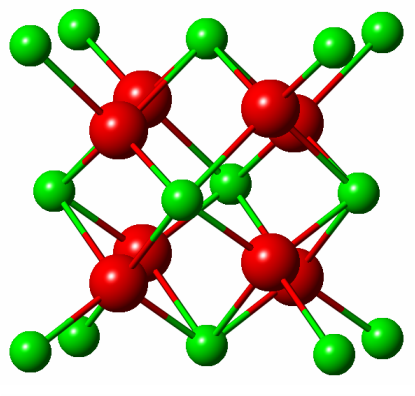

d)

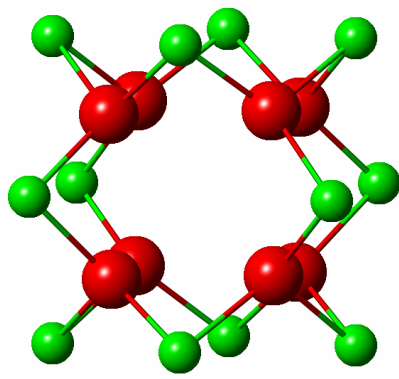

b)

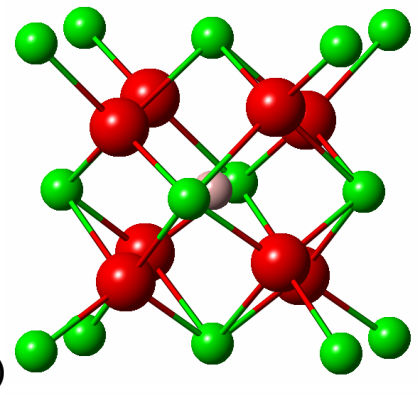

e)

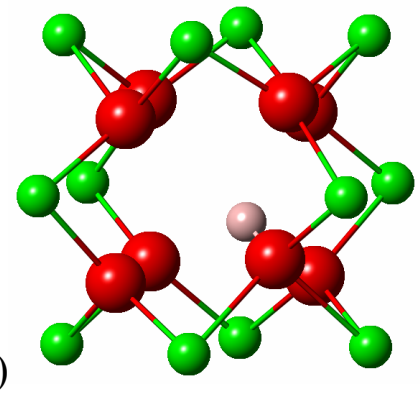

c)

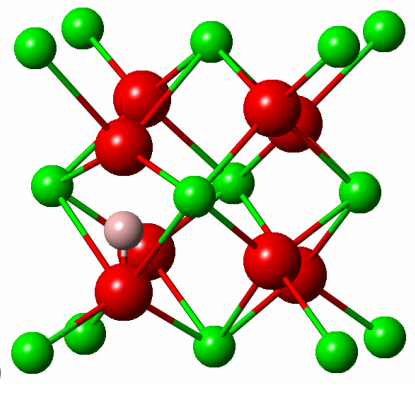

Figure 2. Tetragonal $\mathrm{ZrO}_{2}$ : a) perfect lattice, b) $\mathrm{H}$ in the center of the interstitial space, c) $\mathrm{H}$ bound to an oxygen atom, d) $V_{\mathrm{Zr}}$, and e) $\mathrm{H}$ in $V_{\mathrm{Zr}}$. Sphere designation is the same as in figure 1.

In the case of $V_{\mathrm{Zr}}$-related defects we also calculated positron characteristics. Positron calculations were performed using a real space method, as implemented in the atomic superposition (ATSUP) method [13] using the relaxed atomic configurations obtained with the VASP code. Two approaches were employed: (i) atomic densities and Coulomb potentials were superimposed in order to construct the positron potential that is used to solve the positron Schrödinger equation (ATSUP calculations) and (ii) the valence electron density and Coulomb potential from VASP calculations were combined with those for core electrons obtained from their atomic superposition (ATSUP+VASP calculations). Supercells used in VASP calculations are rather small, and therefore these supercells were extended by adding atoms at their sides when calculating positron properties. Such added atoms were arranged in the form of the regular $\mathrm{ZrO}_{2}$ lattice. In this way, original VASP 96 atom supercells were extended to 768 atom ones.

In positron calculations, the approach of Boroński and Nieminen [14] to the electronpositron correlations and enhancement factor - with the correction [15] for incomplete positron screening with the high frequency dielectric constant $\varepsilon_{\infty}=4.63$ - was used (referred further to as the $\mathrm{BN}$ approach). For the sake of comparison we also employed the so called gradient correction (GC) scheme introduced by Barbiellini et al. [16]. This scheme was implemented into the positron code in order to enable it in ATSUP+VASP calculations (for pure ATSUP calculations it was implemented already earlier). For this purpose, the gradient of the valence electron density needs to be determined on a three dimensional regular mesh (the gradient of the core electron density is obtained using atomic radial wave functions as before). Numerical differentiation is not a difficult task, but in our case its precision needs to be tuned up to be compatible with remaining parts of the code and after thorough testing we selected a seven point formula [17]. We further refer readers to review [18] dealing with the theoretical background of positron calculations. 


\section{RESULTS AND DISCUSSION}

We shall now present and discuss the results for the studied H-related defects in cubic and tetragonal $\mathrm{ZrO}_{2}$ phases from the viewpoint of structural and positron properties.

\section{$\underline{\text { Structure of defects }}$}

Figure 1 shows studied configurations for cubic zirconia. In addition to the perfect lattice (figure 1a) we examined also an $\mathrm{H}$ atom in the $\mathrm{O}_{8}$ interstitial space (figure $1 \mathrm{~b}$ ). In this case the $\mathrm{H}$ atom remains in the centre of the cubic $\mathrm{O}_{8}$ interstitial space and (outward) relaxations of neighboring $\mathrm{O}$ atoms are very small. When the zirconium vacancy is considered (figure 1c), there are large outward relaxations of surrounding of $\mathrm{O}$ ions. If an $\mathrm{H}$ atom is introduced into the center of $V_{\mathrm{Zr}}$, it remains there after the relaxation though surrounding atoms move slightly back compared to the case without $\mathrm{H}$. If energies of all above discussed configurations are taken into account, the binding energy of an $\mathrm{H}$ atom to $V_{\mathrm{Zr}}$ (with respect to position in $\mathrm{O}_{8}$ ) can be estimated and its value is $2.3 \mathrm{eV}$, which indicates that $\mathrm{H}$ atoms prefer to occupy $\mathrm{V}_{\mathrm{Zr}}$ rather than the interstitial $\mathrm{O}_{8}$ space. If the symmetry constraint is removed for configurations shown in figures $1 \mathrm{~b}$ and $1 \mathrm{~d}$, the $\mathrm{H}$ atom moves in the direction towards one of neighboring $\mathrm{O}$ atoms and, at the same time, the symmetry of the structure is changed (not shown here). These configurations differ from those discussed below for the tetragonal phase and will described elsewhere.

As for the tetragonal phase (figure 2), in calculations we used for simplicity the ratio $c / a=1$ (in reality this ratio is $\sim 1.02$ ). The perfect lattice, shown in figure $2 \mathrm{a}$, is characterized by a zigzag arrangement of oxygen atoms in $\langle 100\rangle$-type directions with the alternating coordinate $z$. Like in the cubic case, an $\mathrm{H}$ atom can be located in the center of the $\mathrm{O}_{8}$ interstitial space (which is now somewhat deformed compared to the cube; see figure $2 b$ ), but there exists a lower energy configuration (figure 2c) which retains tetragonal characteristics. For this configuration, there is an $\mathrm{O}-\mathrm{H}$ pair with its bond oriented along the $c$-axis and the $\mathrm{O}-\mathrm{H}$ distance is $\sim 1 \AA$. The $\mathrm{O}_{8}$ interstitial space is apparently distorted compared to its unperturbed state (figure 2a). The O-H bond orientation along the $c$-axis is quite interesting finding as one would rather expect that this bond will be oriented towards the center of the $\mathrm{O}_{8}$ interstitial space.

Relaxing the $V_{\mathrm{Zr}}$ defect (figure 2d) results in a configuration that is very similar to that shown in figure $1 \mathrm{c}$ for the cubic phase. Namely, the tetragonal distortion is almost missing and the $V_{\mathrm{Zr}}$ surrounding is nearly cubic, though in larger distances from the defect (not shown here) the lattice again exhibits the zigzag arrangement of $\mathrm{O}$ atoms. Such an observation could indicate that zirconium vacancies could help to stabilize (at least locally) the cubic phase, but it is not clear why such vacancies should exist in $\mathrm{ZrO}_{2} / \mathrm{YSZ}$ in large concentrations. If an $\mathrm{H}$ atom is introduced into the center of $V_{\mathrm{Zr}}$, it moves towards a neighboring $\mathrm{O}$ atom during relaxation and creates a bond (its length is again about $1 \AA$ ). The bond is oriented towards the $V_{\mathrm{Zr}}$ center and decreases thereby the $V_{\mathrm{Zr}}$ open volume. Binding energies of $\mathrm{H}$ atoms to $V_{\mathrm{Zr}}$ are $6.2 \mathrm{eV}$ and $5.8 \mathrm{eV}$ for configurations with the $\mathrm{H}$ atom in the center of the $\mathrm{O}_{8}$ interstitial space and its side, respectively. Further details of configurations will be published elsewhere.

\section{$\underline{\text { Positron characteristics }}$}

Positron lifetimes and positron binding energies for studied open volume configurations are listed in table I for both phases. 
Table I. Positron characteristics of studied open volume defects.

\begin{tabular}{|l|c|c|c|c|c|c|c|c|}
\hline & \multicolumn{2}{|c|}{ BN } & \multicolumn{2}{c|}{ GC } & \multicolumn{2}{c|}{ BN } & \multicolumn{2}{|c|}{ GC } \\
\hline Configuration & $\begin{array}{c}\tau \\
(\mathbf{p s})\end{array}$ & $\begin{array}{c}\boldsymbol{E}_{\mathbf{b}} \\
(\mathbf{e V})\end{array}$ & $\begin{array}{c}\tau \\
(\mathbf{p s})\end{array}$ & $\begin{array}{c}\boldsymbol{E}_{\mathbf{b}} \\
(\mathbf{e V})\end{array}$ & $\begin{array}{c}\tau \\
(\mathbf{p s})\end{array}$ & $\begin{array}{c}\boldsymbol{E}_{\mathbf{b}} \\
(\mathbf{e V})\end{array}$ & $\begin{array}{c}\boldsymbol{\tau} \\
(\mathbf{p s})\end{array}$ & $\begin{array}{c}\boldsymbol{E}_{\mathbf{b}} \\
(\mathbf{e V})\end{array}$ \\
\hline \multicolumn{8}{|c|}{ Cubic phase } \\
\hline Bulk & 138 & - & 146 & - & 133 & - & 157 & - \\
\hline$V_{\mathrm{Zr}}$ & 216 & 2.75 & 238 & 2.60 & 220 & 2.81 & 291 & 2.42 \\
\hline$V_{\mathrm{Zr}}+1 \mathrm{H}$ (center) & 169 & 1.76 & 181 & 1.68 & 167 & 1.28 & 199 & 1.31 \\
\hline \multicolumn{8}{|c|}{ Tetragonal phase } \\
\hline Bulk & 138 & - & 146 & - & 133 & - & 158 & - \\
\hline$V_{\mathrm{Zr}}$ & 216 & 2.74 & 238 & 2.59 & 219 & 2.76 & 291 & 2.37 \\
\hline$V_{\mathrm{Zr}}+1 \mathrm{H}$ & 189 & 2.23 & 204 & 2.12 & 189 & 1.76 & 237 & 1.54 \\
\hline \multicolumn{8}{|c|}{ ATSUP } \\
\hline
\end{tabular}

One can see that differences between corresponding positron characteristics for the cubic and tetragonal phase are negligible for the bulk and $V_{\mathrm{Zr}}$ cases. This indicates that positrons are not sensitive to the phase where they annihilate, at least for the two phases investigated. As for the $V_{\mathrm{Zr}}+1 \mathrm{H}$ complex, there are differences between positron characteristics because these complexes differ essentially by the position of the $\mathrm{H}$ atom (see above). We also note that the positron lifetime (binding energy) decreases significantly due to the presence of the $\mathrm{H}$ atom in the zirconium vacancy. The effect of the charge transfer (ATSUP+VASP vs ATSUP calculations) is not very apparent for the $\mathrm{BN}$ scheme in contrast to the GC one. Furthermore, the $\mathrm{GC}$ scheme results in apparently larger lifetimes compared to $\mathrm{BN}$ (like for $\mathrm{ZnO}$ - see ref. [4]). As the bulk positron lifetime in $\mathrm{ZrO}_{2} / \mathrm{YSZ}$ has not been determined from an experiment till now, it is not possible to judge which scheme describes better positron correlations in this material though on the basis of previous investigations of oxides [4,19] we do not expect that experimental bulk lifetime would fall by a large margin outside the interval given by the $\mathrm{BN}$ and $\mathrm{GC}$ values. On the other hand, in the case of the zirconium vacancy the GC lifetime exceeds the BN one by about $70 \mathrm{ps}$ for ATSUP+VASP calculations, which is a too large difference indicating that the $\mathrm{GC}$ scheme might not be appropriate for larger open volumes in $\mathrm{ZrO}_{2}$. In any case, this effect needs to be further investigated. From the experimental viewpoint, the positron lifetime observed for a cubic YSZ single crystal $\left(9 \mathrm{~mol} . \% \mathrm{Y}_{2} \mathrm{O}_{3}\right)$, as reported in ref. [9], amounts to $175 \mathrm{ps}$ (saturated trapping). This number falls into the interval between calculated ranges of the bulk and $V_{\mathrm{Zr}}$ positron lifetimes. This suggests that the observed lifetime could originate from $V_{\mathrm{Zr}}$-hydrogen complexes, even if uncertainties in the theoretical description are taken into account.

\section{CONCLUSIONS}

We have studied hydrogen in several positions in $\mathrm{ZrO}_{2}$ cubic and tetragonal lattices. The most stable $\mathrm{H}$ positions are not in the center of interstitial space or $V_{\mathrm{Zr}}$, but $\mathrm{H}$ atoms rather tend to create bonds with neighboring $\mathrm{O}$ atoms. The study is not yet complete and further calculations are in progress to examine other possible defect configurations involving also more than one $\mathrm{H}$ atom (including $\mathrm{H}_{2}$ molecule). It was also shown that hydrogen may shorten positron lifetime of 
zirconium vacancies in $\mathrm{ZrO}_{2}$, which could eventually explain experimental positron lifetime observed in YSZ single crystals. This effect has been proven to happen in $\mathrm{ZnO}$ [4].

\section{ACKNOWLEDGMENTS}

We are grateful to M. J. Puska for his ATSUP code that served as a basis for further developments. Financial support of the Academy of Sciences of the Czech Republic (project KJB101120906) is appreciated. This work was further supported by the Ministry of Schools, Youths and Sports of the Czech Republic through the research plan MSM 0021620834 and the project COST OC 165. The support provided by the computational facility LUNA (Academy of Sciences of the Czech Republic, VUMS Computers) is also acknowledged.

\section{REFERENCES}

1. Positron Spectroscopy of Solids, edited by A. Dupasquier and A. P. Mills, Jr. (IOS, Amsterdam, 1995).

2. X. Guo and Z. Wang, J. Eur. Ceram. Soc. 18, 237 (1998).

3. O. Melikhova, J. Kuriplach, J. Cizek, I. Prochazka, and G. Brauer, Mater. Sci. Forum 607, 125 (2009).

4. G. Brauer, W. Anwand, D. Grambole, J. Grenzer, W. Skorupa, J. Cizek, J. Kuriplach, I. Prochazka, C. C. Ling, C. K. So, D. Schulz, and D. Klimm, Phys. Rev. B 79, 115212 (2009).

5. D. Grambole et al., to be published.

6. C. G. Van de Walle, Phys. Rev. Lett. 85, 1012 (2000).

7. A. Janotti and C. G. Van de Walle, Nature Mater. 6, 44 (2007).

8. J. Kuriplach, G. Brauer, O. Melikhova, J. Cizek, I. Prochazka, and W. Anwand, 2009 MRS Fall Meeting Proceedings (Symposium H).

9. O. Melikhova, J. Kuriplach, J. Cizek, I. Prochazka, G. Brauer, and W. Anwand, submitted to J. Phys.: Conf. Series; Advanced Science Research Symposium, Tokai, Japan, November 2009.

10. G. Kresse and J. Hafner, Phys. Rev. B 47, 558 (1993), ibid. 49, 14251 (1994); G. Kresse and J. Furthmüller, Comput. Mater. Sci. 6, 15 (1996); Phys. Rev. B 54, 11169 (1996).

11. G. Kresse and J. Hafner, J. Phys.: Condens. Matter 6, 8245 (1994); G. Kresse and D. Joubert, Phys. Rev. B 59, 1758 (1999).

12. S. Ostanin, E. Salamatov, A. J. Craven, D. W. McComb, and D. Vlachos, Phys. Rev. B 66, 1132105 (2002).

13. M. J. Puska and R. M. Nieminen, J. Phys. F: Met. Phys. 13, 333 (1983); A. P. Seitsonen, M. J. Puska, and R. M. Nieminen, Phys. Rev. B 51, 14057 (1995).

14. E. Boroński and R. M. Nieminen, Phys. Rev. B 34, 3820 (1986).

15. M. J. Puska, S. Mäkinen, M. Manninen, and R. M. Nieminen, Phys. Rev. B 39, 7666 (1989).

16. B. Barbiellini, M. J. Puska, T. Torsti, and R. M. Nieminen, Phys. Rev. B 51, 7341 (1995).

17. J. H. Mathews, Int. J. Math. Edu. Sci. Tech. 34, 280 (2003).

18. M. J. Puska and R. M. Nieminen, Rev. Mod. Phys. 66, 841 (1994).

19. J. Kuriplach, O. Melikhova, and G. Brauer, Rad. Phys. Chem. 76, 101 (2007). 